\title{
Maternal prenatal iron status and tissue organization in the neonatal brain
}

\author{
Catherine Monk ${ }^{1,2,3}$, Michael K. Georgieff', Dongrong X ${ }^{5}{ }^{5}$, Xuejun Hao ${ }^{5}$, Ravi Bansal ${ }^{6}$, Hanna Gustafsson', \\ Julie Spicer ${ }^{1}$ and Bradley S. Peterson ${ }^{6,7}$
}

BACKGROUND: Children prenatally exposed to inadequate iron have poorer motor and neurocognitive development. No prior study to our knowledge has assessed the influence of maternal prenatal iron intake on newborn brain tissue organization in full-term infants.

METHODS: Third trimester daily iron intake was obtained using the Automated Self-Administered 24-h Dietary Recall with $n=40$ healthy pregnant adolescents (aged 14-19y). Cord blood ferritin was collected in a subsample $(n=16)$. Newborn (mean $=39$ gestational weeks at birth; range 37-41) magnetic resonance imaging scans were acquired on a 3.0 Tesla MR Scanner. Diffusion Tensor Imaging (DTI) slices were acquired to measure the directional diffusion of water indexed by fractional anisotropy (FA).

RESULTS: Reported iron intake was inversely associated with newborn FA values $(P \leq 0.0001)$ predominantly in cortical gray matter. FA findings were similar using cord blood ferritin values. CONCLUSION: Higher maternal prenatal iron intake accentuates, and lower intake attenuates, the normal age-related decline in FA values in gray matter, perhaps representing increasing dendritic arborization and synapse formation with higher iron intake. These DTI results suggest that typical variation in maternal iron outside the scope of standard clinical surveillance exerts subtle effects on infant brain development.

ron, an abundant micronutrient, is essential for optimal brain growth in utero. Nevertheless, $35-58 \%$ of healthy women have some degree of iron deficiency (1), with rates even higher during pregnancy (2), even in well-resourced countries such as the United States (3). Worldwide, 50\% of pregnant women are anemic (2). Iron deficiency for the fetus most often is found to occur as a consequence of severe maternal iron deficiency anemia, frequently due to poor compliance with maternal iron supplementation, but can occur with other gestational conditions (e.g., diabetes, hypertension, cigarette smoking) (4).
Sufficient iron availability in the developing fetal brain is essential to support neuronal and glial energy metabolism, dendritic arborization, synaptogenesis, neurotransmitter synthesis, and emerging myelination (5). Animal models demonstrate that prenatal brain iron deficiency leads to impaired hippocampal functioning and neurochemical abnormalities (6), which in turn adversely affect learning and memory (7). Fetal and early neonatal iron deficiency in rodents produces less linear growth of dendrites and disordered dendritic branches in the hippocampus that persist into adulthood $(6,8)$. Animal models have shown that perinatal iron deficiency also is associated with delayed myelination of subcortical white matter (9), and decreased myelin content in cerebellar white matter as assessed histopathologically (10). This latter study of cerebellar white matter modeled human gestational iron deficiency most closely in rodents by continuing it only until testing at postnatal day 11, the developmental equivalent of term human birth.

Consistent with these findings from animal studies, newborns with a low iron profile have poorer auditory recognition memory at birth (11), poorer explicit (delayed imitation recall) memory performance until the age of $4 \mathrm{y}$ (12), and poorer performance on tests of general motor and neurocognitive development (13). Children born with low cord blood ferritin, the major storage form of iron, have greater academic problems in school and impaired immediate recall memory, delayed recall memory, and working memory for their age (for a summary of findings, see ref. 7). Infants with cord ferritin concentrations in the lowest quartile showed by the age of $5 \mathrm{y}$ less developed language abilities, fine and gross motor movement, and attention (14).

To date, only two studies $(11,15)$ have examined maternal prenatal iron status as a function of iron accretion (as indexed by cord blood ferritin) in relation to newborn brain characteristics in those born full term. Both studies used electrophysiological recordings and suggested that deficient fetalneonatal iron status is associated with abnormal newborn

\footnotetext{
'Division of Behavioral Medicine, Department of Psychiatry, Columbia University Medical Center, New York, New York; ${ }^{2}$ Department of Obstetrics and Gynecology, Columbia University Medical Center, New York, New York; ${ }^{3}$ New York State Psychiatric Institute, New York, New York; ${ }^{4}$ Division of Pediatric Neonatology, Department of Pediatrics,

University of Minnesota Medical School, Minneapolis, Minnesota; ${ }^{5}$ Division of Epidemiology, Department of Psychiatry, New York State Psychiatric Institute, New York, New York; ${ }^{6}$ Department of Pediatrics, Children's Hospital Los Angeles and the University of Southern California, Los Angeles, California; ${ }^{7}$ Division of Child and Adolescent Psychiatry, Keck School of Medicine, University of Southern California, Los Angeles, California. Correspondence: Catherine Monk (cem31@cumc.columbia.edu)

Received 24 April 2015; accepted 9 September 2015; advance online publication 23 December 2015. doi:10.1038/pr.2015.248
} 
brain development in cortical gray matter and axonal pathways. In one study of 23 newborns (38-42 wk postmenstrual age (PMA)), 9 infants with suspected brain iron deficiency (based on cord ferritin $\leq 34 \mu \mathrm{g} / \mathrm{l}$ ) had shorter latencies to the peak components of an evoked response potential in response to auditory stimuli (11), suggesting either altered transmission time in the nervous system that may be related to the degree of myelination or a lack of sustained neural activity representing differences in the formation and function of axons, dendrites, and synapses. In contrast, in a study of 45 newborns (gestational age at birth $>35 \mathrm{wk}$, tested $24-48 \mathrm{~h}$ after birth), 12 with latent iron deficiency (based on cord ferritin $<37 \mu \mathrm{g} / \mathrm{l}$ ) had prolonged interpeak latencies in an auditory brainstem evoked responses (15), indicating the presence of slowed nerve conduction velocity, which was interpreted as possibly representing reduced myelination of the auditory nerve.

Diffusion Tensor Imaging (DTI) measures diffusion of water. The degree of its directionality, quantified as fractional anisotropy (FA), represents the organization and integrity of brain tissue. DTI studies in newborn brains have shown that FA within white matter increases with gestational age $(16,17)$. Although one prior DTI study detected variation in white matter development related to diet in infancy (18), to date no DTI study to our knowledge has assessed the influence of maternal prenatal iron intake on DTI-based measures of newborn brain development. Iron deficiency induces disturbances in brain development that could influence FA independently in white and gray matter.

In this study, we used DTI to evaluate newborn brain tissue organization in relation to maternal prenatal iron status so as to determine whether and how maternal iron intake affects newborn brain structure. Between 34 and 36 gestational weeks, maternal reports of iron intake were obtained via self-report; for a subsample, cord blood ferritin was collected at birth. During the third trimester, the fetus accumulates iron at a dramatic average rate of $1.35 \mathrm{mg} / \mathrm{kg}$ of fetal weight, maintaining an average iron content of $75 \mathrm{mg} / \mathrm{kg}$ of body weight during this period of rapid brain development $(4,19)$. We recruited pregnant, generally healthy adolescents (aged 14-19 y) because they are at high risk for iron deficiency due to the demands of maternal growth in addition to those of the developing fetus (20).

\section{RESULTS}

Forty young pregnant women and neonates completed the study. The sample was predominantly Latina, and the infants were born at term and at typical weight (Table 1). However, because gestational age at birth and PMA at scan varied across infants and are known to influence FA values, these variables were included as covariates in all analyses.

Based on third trimester responses to the web-based Automated Self-Administered 24-hour Dietary Recall (ASA24), as well as micronutrient estimates from prenatal vitamins, participants reported an average daily intake of 2,443 calories (1214.54 SD) that included $40.59 \mathrm{mg}$ of iron (16.74 SD) (Table 2). However, 20\% were getting less than the US
Table 1. Demographic information on the subjects

\begin{tabular}{|c|c|c|c|c|c|}
\hline Variables & $N$ & Mean/\% & SD & Min. & Max. \\
\hline \multicolumn{6}{|l|}{ Maternal } \\
\hline Age & 40 & 18.23 & 1.37 & 14 & 20 \\
\hline $\begin{array}{l}\text { Weight before } \\
\text { pregnancy (lbs) }\end{array}$ & 40 & 142.23 & 38.77 & 85 & 279 \\
\hline $\begin{array}{l}\text { Weight gain during } \\
\text { pregnancy (lbs) }\end{array}$ & 38 & 27.83 & 12.70 & 9 & 47 \\
\hline Income $<\$ 25,000$ & 40 & $80 \%$ & & & \\
\hline \multicolumn{6}{|l|}{ Ethnicity } \\
\hline Hispanic & 38 & 95 & & & \\
\hline Non-Hispanic & 2 & 5 & & & \\
\hline \multicolumn{6}{|l|}{ C section } \\
\hline No & 33 & 83 & & & \\
\hline Yes & 7 & 17 & & & \\
\hline \multicolumn{6}{|l|}{ Pregnancy complications ${ }^{a}$} \\
\hline None & 39 & & & & \\
\hline 1,2 or 3 complications & 1 & & & & \\
\hline 5 complications & 0 & & & & \\
\hline \multicolumn{6}{|l|}{ Neonate } \\
\hline Birth weight (g) & 40 & 3226.53 & 450.48 & 2475 & 4315 \\
\hline Gestational age at birth (wk) & 39 & 39.26 & 1.12 & 36.71 & 41.00 \\
\hline Postmenstrual age at scan (wk) & 40 & 41.98 & 1.41 & 38.71 & 44.57 \\
\hline $\begin{array}{l}\text { \# of days after birth scan } \\
\text { occurred (d) }\end{array}$ & 40 & 19.63 & 8.31 & 4 & 37 \\
\hline \multicolumn{6}{|l|}{ Sex } \\
\hline Male & 25 & 63 & & & \\
\hline Female & 15 & 37 & & & \\
\hline $\begin{array}{l}\text { Infant feeding: breast- and } \\
\text { bottle-feeding }\end{array}$ & 33 & $76 \%$ & & & \\
\hline
\end{tabular}

Recommended Daily Allowance (RDA) (21) of $27 \mathrm{mg}$ iron for pregnant women. Moreover, although the majority of participants endorsed taking prenatal vitamins, $58 \%$ had blood hemoglobin levels below normal $(<11 \mathrm{~g} / \mathrm{l}$ in the third trimester), and $14 \%$ were at the criterion for mild anemia $(<10 \mathrm{~g} / \mathrm{l})(2) ; 58 \%$ had low hematocrit values (lower than 33\% red blood cells to plasma volume) (22). For the subsample $(n=16)$ for whom we had cord blood serum ferritin concentrations, 3 had significantly elevated ferritin concentrations $(>370 \mu \mathrm{g} / \mathrm{l})$, indicating probable perinatal inflammation (19); as an a priori decision, all analyses are reported excluding these subjects though we ran analyses including them and the results did not differ. Two infants had ferritin values in the lowest quartile $(<76 \mu \mathrm{g} / \mathrm{l})$ in one classification (4) and a third infant was close to it, at 77 $\mu \mathrm{g} / \mathrm{l}(14)$; four were in this category based on another classification $(<82 \mu \mathrm{g} / \mathrm{l})(4)$. Cord blood ferritin in the lowest quartile has been associated with neurologic abnormalities in multiple 


\section{Articles | Monketal.}

studies $(11,14)$. Total third trimester maternal reported enteral iron intake correlated significantly with cord blood ferritin ( $r=0.57, P \leq 0.05, n=13)$, supporting the validity of the maternal reports of dietary intake.

To determine if our data showed typical developmental trends for FA, we first assessed the voxelwise correlations of infant PMA with FA values, controlling for gestational age at birth, sex, weight at birth and maternal iron intake (based on self-report). Neonatal age at the time of scan correlated positively and significantly with FA values $(P \leq 0.0001)$, with significant correlations clustering in major axonal pathways of the brain, including the anterior region of the corona radiata, splenium of the corpus callosum, internal capsule, longitudinal fasciculus, optic radiation, and superior region of the

Table 2. Iron status: maternal third trimester plasma and neonatal cord blood

\begin{tabular}{lccccc}
\hline Variables & $N$ & Mean/\% & SD & Min. & Max. \\
\hline Maternal & & & & & \\
$\quad \begin{array}{l}\text { Average total daily calories } \\
\text { (cal) }\end{array}$ & 38 & 2443.30 & 1214.54 & 159.8 & 5358.43 \\
Average total daily iron (mg) & 40 & 40.59 & 16.74 & 5.96 & 83.34 \\
$\begin{array}{l}\text { Prenatal vitamins and/or iron } \\
\text { supplement }\end{array}$ & 40 & & & & \\
$\quad$ No & 2 & 5 & & & \\
$\quad$ Yes & 38 & 95 & & & \\
Hbc (g/l) & 36 & 108.1 & 9.5 & 93 & 132 \\
Hematocrit & 36 & 32.42 & 2.56 & 27.9 & 37.7 \\
MCV (fl) & 36 & 86.19 & 6.39 & 64.4 & 101.6 \\
Neonate (cord blood) & & & & & \\
Ferritin ( $\mu \mathrm{g} / \mathrm{l})$ & 16 & 215.44 & 171.44 & 18 & 575 \\
Iron (mg/d) & 16 & 137 & 54.91 & 44 & 248 \\
\hline
\end{tabular}

$\mathrm{Hb}$, hemoglobin; MCV, mean corpuscular volume.

asubject with very low calories had not yet eaten that day at time of study session. ${ }^{\text {bThee }}$ subjects were missing third trimester ASA24 (Automated Self-Administered 24-hour Dietary Recall) data or had outlier values; iron values were carried forward from prior sessions. Three subjects were missing 3 rd trimester $\mathrm{Hb}$ values due to receiving prenatal care outside of CUMC (Columbia University Medical Center) and one because that data point was missing from CUMC medical records. Maternal iron intake was not significantly correlated with total calorie consumption. corona radiata (Figure 1). Inverse correlations of PMA with FA were scattered diffusely throughout the brain, making it difficult to assign findings definitively either to specific cortical gray matter regions or to specific, nearby axonal pathways. Nevertheless, significant inverse correlations of PMA with FA $(P \leq 0.0001)$ were located preferentially near the surface of the brain, presumably in cortical gray matter, somewhat more concentrated in frontal regions, but present in posterior brain regions as well (Figure 1).

Significant inverse correlations of total maternal reported iron intake with FA values were detected diffusely throughout the brain, without clear preferential clustering in specific regions or tissue type $(P \leq 0.0001)$ (Figure 2$)$. A predominance of these inverse associations were located toward the periphery of the brain, clearly in cortical gray matter, whereas a minority of others were located in areas consistent with axonal pathways. For example, as seen in Figure 2, scatterplots for correlations in representative voxels are as follows: thalamus, $r=-0.55$; occipital cortex $r=-0.53$; parieto-occipital cortex, $r=-0.47$; temporoparietal cortex, $r=-0.60$; all $P$ s $<0.001$. Overall, these inverse associations indicated that lower maternal iron intake was associated with higher FA values while covarying for PMA at the time of the scan, gestational age at birth, sex and birth weight. Correlations were similar in the subsample of 13 infants for whom cord blood ferritin values were available (Figure 2), supporting the validity of our findings using maternal report of iron intake.

\section{DISCUSSION}

This is the first study to use DTI to associate maternal prenatal iron intake to differences in newborn brain tissue organization. Maternal iron intake correlated inversely with FA values predominantly in cortical gray matter but also, to a lesser extent, in major axonal pathways. These findings were validated in a subsample of infants for whom cord ferritin levels were available. The correlations of maternal iron status with DTI-based measures of brain tissue organization were detected in the newborn infants of a sample of healthy pregnant adolescents who were adhering to prenatal care and across a range of iron intake. Twenty percent were receiving less than the RDA for iron, and $14 \%$ met clinical criteria for mild anemia. For the subsample

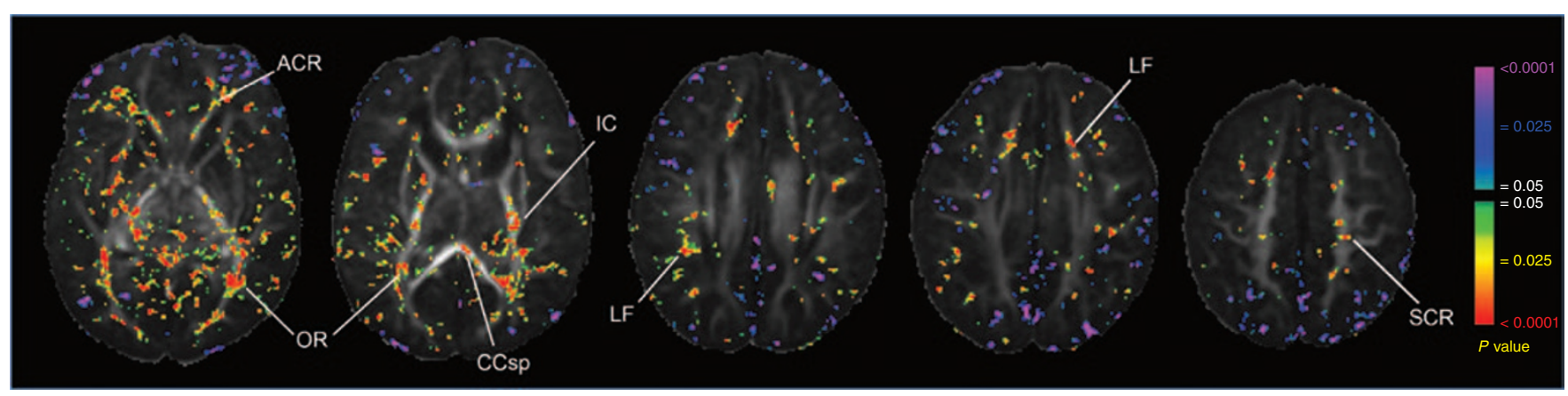

Figure 1. Neonatal age at the time of scan correlated positively with fractional anisotropy (FA) values $(P \leq 0.0001)$ in major and minor axonal pathways, including anterior region of the corona radiata (ACR), splenium of the corpus callosum (CCsp), internal capsule (IC), longitudinal fasciculus (LF), optic radiation (OR), and superior region of the corona radiata (SCR). Significant inverse correlations of postmenstrual age with $\mathrm{FA}(P \leq 0.0001)$ were located in the frontal and posterior regions of the brain. 


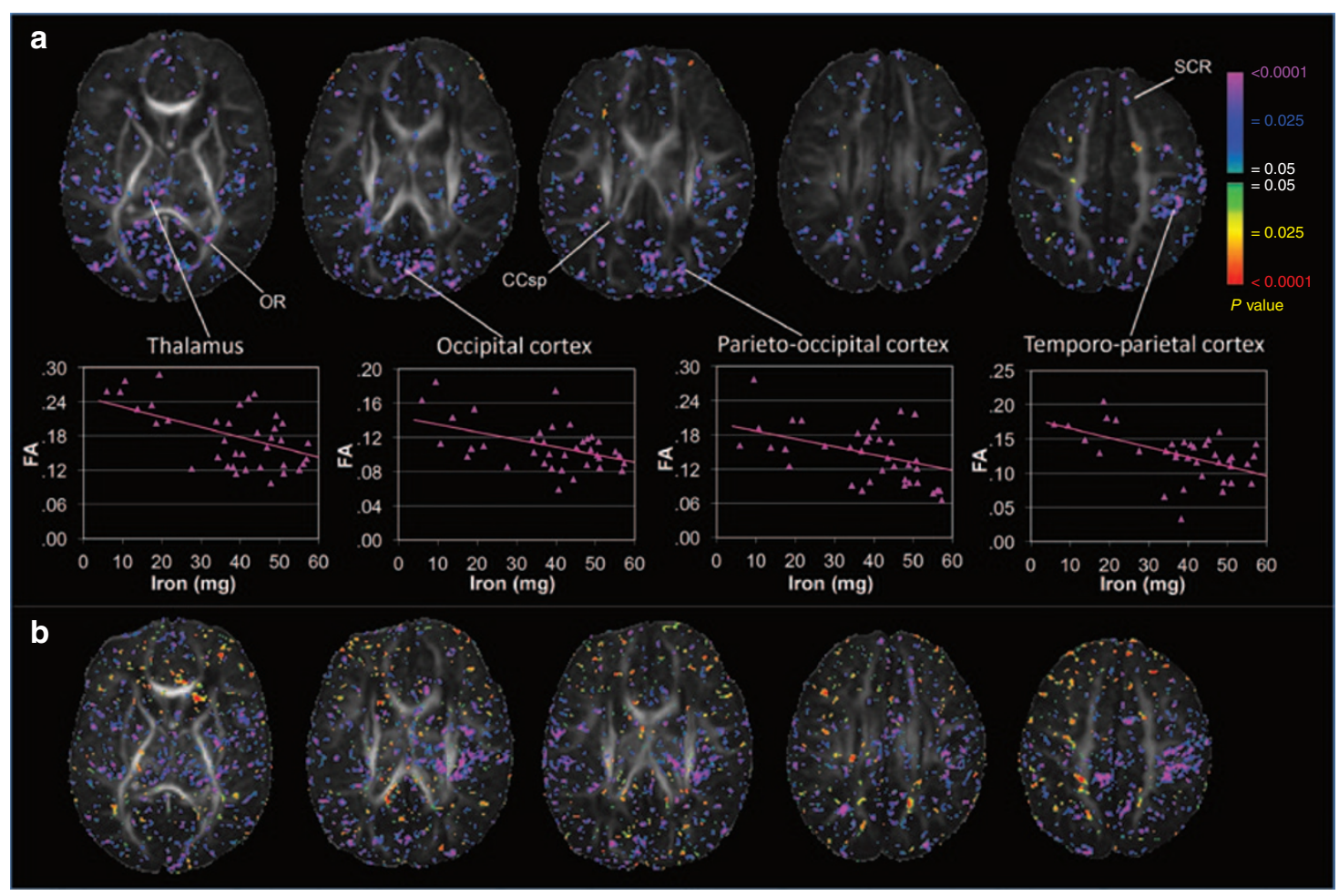

Figure 2. Significant inverse correlations of total maternal reported iron intake with fractional anisotropy (FA) values $(P \leq 0.0001)$ were detected diffusely throughout the brain within gray matter. (A) Scatterplots for randomly selected voxels suggest that the inverse correlations were not driven by outliers. Inverse correlations were observed in white matter tracts, including splenium of the corpus callosum (CCsp), optic radiation (OR), and superior region of the corona radiata (SCR). (B) Similar significant correlations between cord blood ferritin and FA values were found $(P \leq 0.0001)$, supporting the validity of our findings with maternal report of iron intake.

with cord ferritin values, three had the lowest quartile ferritin values $(\leq 77 \mu \mathrm{g} / \mathrm{l})(19 \%)$ according to common standards (14), including one who was at the level indicating suspected brain iron deficiency $(\leq 34 \mu \mathrm{g} / \mathrm{l})(11)$. A prior study with pregnant adolescents identified $29 \%$ as anemic at birth, with $11 \%$ of the newborns having ferritin levels as low as $\leq 34 \mu \mathrm{g} / \mathrm{l}$ (20). None of our participants were recognized clinically as having lifestyle habits that would interfere with their infant's brain development.

FA in axonal pathways increases with age in both children (23) and newborns $(16,17)$, presumably as a consequence of declining water content, the formation of myelin, and development of new barriers to water mobility, such as cell membranes and organelles (24). Studies with preterm infants (25) and nonhuman primates (26) have shown that FA decreases over time in cortical gray matter as a consequence of a maturational increase in cellular and synaptic complexity, elongation of dendrites (25), and increase in arborization of axons and dendrites (27). Consistent with these prior reports in animals and humans is our finding that FA increased with age in newborn infants within clusters located within major axonal pathways bilaterally, such as the anterior region of the corona radiata, splenium of the corpus callosum, and the optic radiations. Conversely, FA declined with age primarily within cortical gray matter, perhaps somewhat more concentrated in frontal regions, but also in posterior brain areas.

Within the context of these expected age-related effects on FA values, we found that maternal iron intake correlated inversely with FA at locations scattered throughout the brain, mostly in cortical gray matter and perhaps, more rarely, within axonal pathways. The inverse correlations in cortical gray matter suggest that higher maternal prenatal iron intake may accentuate, and that lower iron intake may attenuate, the normal agerelated decline in FA values in gray matter. These findings are consistent with our expectations. Cortical tissue consists primarily of nerve cell bodies and neuropil (arborized dendrites, axons, and synapses), which tend not to have directional coherence within a volume of tissue as large as a DTI voxel. Greater degrees of axonal and dendritic arborization reduce directional coherence (26), thereby reducing FA values (25). Consistent with these prior findings, our group has shown elsewhere that a greater density of neural tissue in human cortical gray matter, as indexed by levels of $\mathrm{N}$-acetyl aspartate measured using MR spectroscopy, which presumably accompanies an increase in the complexity of neuropil, is associated significantly with lower FA values (27). Cortical neuropil develops dramatically during the third trimester of human gestation and early postnatal life, when dendritic arborization and synaptogenesis accelerate to produce a thickening of the cortical mantle. Synaptogenesis peaks at this time, with nearly 40,000 new synapses formed each second (28). In animal models, perinatal iron deficiency is associated with truncated and simplified dendritic arbors in the hippocampus (7). Behavior in these iron-depleted animals is consistent with these developmental alterations in brain structure, as they are impaired in recognition and procedural 


\section{Articles | Monketal.}

memory. Iron deficiency from late gestation through 2-3 y old in human children is also associated with deficits in memory and learning (reviewed in refs. 7,29).

Within axonal pathways and regions of future white matter in our infants (axons are not yet myelinated at birth and therefore these regions are not "white"), even the infrequent presence of inverse correlations of maternal iron intake with infant FA values at birth may at first seem somewhat counterintuitive-i.e., higher iron intake attenuates the normal, age-related increase in infant FA values in axonal pathways, whereas lower iron intake accentuates it. Several prior studies in animals $(9,10,30)$ suggest that lower perinatal iron intake is associated with delayed white matter development. Our seemingly discrepant findings compared with these prior studies could have one or more explanations. First, the age of exposure to inadequate iron and of assessment: two of the three animal studies included the influence of postnatal as well as gestational iron deficiency $(9,30)$ and tested offspring at postnatal day 21 , beyond the time frame considered comparable to the newborn period (postnatal day 7-10). The other animal study (10) used histopathology to identify decreased myelin content in the cerebellar white matter at postnatal day 11. Second, myelin content of the human newborn brain is very low at birth and is entirely absent in most axonal pathways (28); consequently, regional and betweeninfant variation in myelin is so low as to preclude substantial contribution of myelin to neonatal FA values.

Alternatively, the inverse association between maternal iron intake and FA values in axonal pathways could indicate that lower maternal iron intake is associated with greater axonal density and tissue organization. In other words, lower iron levels could accentuate the age-related increase in FA and, by extension, tissue organization in newborn infants. This interpretation of our findings would suggest that inadequate prenatal iron could accelerate brain maturation in axonal pathways, a possibility that is consistent with emerging research on the developmental influences of low iron and other nonoptimal prenatal experiences. For example, iron deficiency in one of our prior studies with newborns was associated with shorter latencies to peak evoked response potential components during presentation of acoustic stimuli (11). In a rodent model of perinatal iron deficiency, furthermore, inadequate iron was associated with significantly lower auditory brainstem response latencies, suggesting the presence of abnormally rapid signal conduction in iron deficiency. Of particular relevance to our DTI findings, this decreased auditory brainstem response latency was associated with the presence of larger axon diameters (31). Compared to healthy, term-born infants, preterm babies with Intrauterine Growth Restriction (50\% of whom have ferritin levels below the fifth percentile (32)) had higher FA values at 12 mo of age in frontal white matter regions (33). As noted in these studies (33), FA as an index of "increased" maturation in white matter does not necessarily mean "better"; instead, the association of higher FA values with preterm birth in these studies, or with lower maternal iron intake in our study, may represent an aberrant maturational trajectory for axonal pathways and future white matter. This interpretation as it pertains to our findings of maternal iron intake are offered cautiously, however, as our data are preliminary and clearly require replication.

From a public health perspective, these iron-related effects in the newborn brain were identified in a relatively homogenous sample of healthy pregnant women, albeit adolescents, receiving prenatal care, the majority of whom reported taking prenatal vitamins, and none of whom had medical illnesses. Despite this adherence to good prenatal health practices, $20 \%$ were receiving less than the US RDA of iron for pregnant women based on self-report, $14 \%$ had blood hemoglobin levels that met criterion for mild anemia (2), and 19\% of their babies had cord blood ferritin levels $(\leq 76-77 \mu \mathrm{g} / \mathrm{l})$ that have been associated with impaired language and motor development at $5 \mathrm{y}$ of age (14). In this context, our findings showing associations between maternal prenatal iron levels and tissue organization in the newborn brain that complements extensive evidence implicating low maternal prenatal iron availability in compromising child neurodevelopmental outcomes (2) underscore the likelihood that the typical variation in maternal iron stores outside the scope of standard clinical surveillance exerts subtle effects on infant brain development. Of note, our scatterplots (Figure 2) provide some evidence for an incremental, dose-response association between maternal iron status and newborn brain tissue organization, with little evidence of a threshold effect. While necessitating further study and corroborative findings, this finding indicates the potential significance for child outcomes of even modest alterations in maternal dietary health.

This study has several limitations. First, because of the highly technical nature of the brain assessment, the sample size was relatively small and limited our statistical power to detect real effects. Second, we cannot assert true developmental effects relating maternal iron intake to newborn brain characteristics in a cross-sectional study. We also do not know the cognitive and behavioral correlates of our iron-related FA values and newborn brain tissue organization or whether these results will generalize to pregnant adult women and their newborns. Finally, FA interpretation presents challenges in studies of newborn infants without knowledge of the effects of changing intra- and extracellular water concentration with age on FA values (34) and how variations in maternal iron intake can affect those changing water concentrations.

When compromised, the early neurocognitive development of children constrains their psychosocial adaptation and their academic and later occupational trajectories. Clinical, epidemiologic, and basic science research suggests that experiences common in pregnancy, such as diet, distress, environmental pollutants, and exercise, significantly influence brain development in children. Our imaging findings add brain-based assessments to the growing evidence that common inadequacies in maternal nutrition influence developmental trajectories in children, even before birth.

\section{METHODS}

\section{Subjects}

Healthy, nulliparous pregnant adolescents (aged 14-19 y; by the time of the newborn session, some of the participants were aged $20 \mathrm{y}$ ) who were actively engaged in prenatal care were recruited through the 
Departments of Obstetrics and Gynecology at Columbia University Medical Center (CUMC) and Weill Cornell Medical College, and flyers posted in the CUMC vicinity for a longitudinal pregnancy study. Participants gave informed consent, and procedures were in accordance with the Institutional Review Board of the New York State Psychiatric Institute/CUMC. Participants were excluded if they acknowledged smoking or use of recreational drugs, use of medications with an effect on cardiovascular function, or lacked fluency in English.

\section{Study Visits}

Participants were seen during three study visits during pregnancy, cord blood collection occurred at birth, and the infant magnetic resonance imaging (MRI) sessions were conducted within, on average, $2.8 \mathrm{wk}$ (or $19.63 \mathrm{~d}$ ) of birth. This report concerns the third visit in the third trimester (34-36th wk), collection of biological samples at birth, and the MRI scan.

Iron status self-report. During the third pregnancy visit, nutrition information was acquired through the ASA24. The ASA24 is an internet-based questionnaire provided by the National Cancer Institute. The ASA24 is a detailed questionnaire that asks the participant to recall food intake over the preceding $24 \mathrm{~h}$ using detailed probes and portion-size food images. ASA24 estimates relative micronutrient levels using three databases: the USDA's Food and Nutrient Database for Dietary Surveys (FNDDS), the USDA's MyPyramid Equivalents Database (MPED), and the USDA's Center for Nutrition Policy and Promotion's MPED Addendum. For three participants, the ASA24 iron intake variable was taken from the second $(n=1)$ or first $(n=2)$ session because the value was missing or an outlier $(>10 \times$ the SD of the mean) at the third session. None of these participants' iron values ever were $<27 \mathrm{mg}$, the RDA for iron intake. DTI analyses were run excluding these participants' with replaced values, and the results were unchanged.

Newborn and maternal health characteristics. Data were obtained from the medical record.

Ferritin assay. Umbilical cord blood was obtained for evaluation of serum ferritin concentrations, determined in duplicate by chemiluminescent immunoassay (Beckman Coulter, Brea, CA). Repeatability precision is $<8 \%$ coefficient of variation and within laboratory precision is $<11 \%$ coefficient of variation.

DTI pulse sequence. MRI scans were performed on a 3.0 Tesla MR Scanner (GE Health Care, Milwaukee, WI) using an 8-channel head coil. DTI slices were acquired in an axial oblique orientation parallel to the anterior-posterior commissure line using single-shot echo planar DTI imaging sequence, with repetition time $=13,925 \mathrm{~ms}$, echo time $=\sim 74 \mathrm{~ms}$, field of view $=19 \times 19 \mathrm{~cm}^{2}$, flip $=90^{\circ}$, acquisition matrix $=132 \times 128($ acceleration factor $=2)$ zero-padded to $256 \times 256$, for 60 oblique axial slices positioned parallel to the anterior-posterior commissure line, slices thickness $=2.0 \mathrm{~mm}$. We acquired 3 baseline images with $b=0 \mathrm{~s} / \mathrm{mm}^{2}$, and 11 diffusion-weighted images at $b=600 \mathrm{~s} / \mathrm{mm}^{2}$ with diffusion gradients applied in 11 directions sampling 3D space uniformly.

\section{Data Analysis}

We corrected magnetic field inhomogeneities, eddy current distortions, and head motion using the FSL toolbox (35). We then computed the diffusion tensor at each voxel by fitting an ellipsoid to the diffusion-weighted imaging data acquired along 11 gradient directions and 3 baseline images using a Levenberg-Marquardt algorithm to achieve a robust nonlinear least squares fit, while constraining the diffusion tensor to be positive definite (36). FA maps generated from the diffusion tensor model were then spatially normalized to the template brain using a rigid body similarity transformation, followed by a nonlinear warping using a method based on fluid dynamics (37).

\section{Statistical Analysis}

Preliminary analyses were performed using SPSS to characterize sample demographics and mean values for iron status. Correlations of FA values with either PMA at the time of scan (while covarying for maternal iron intake sex, gestational age at birth, birth weight) or with iron indices (while covarying for PMA, sex, gestational age at birth and birth weight) were assessed using multiple linear regression applied voxelwise throughout the brain.

We used the False Discovery Rate (FDR) to control for false positives when testing statistical hypothesis across all voxels in the brain within the DTI dataset. FDR generates a significance level $\alpha$ for voxel-level $P$ values such that among all voxels with $P$ values less than $\alpha$ only a specified proportion $q$ are false positive. The proportion $q$ is termed the FDR among the rejected null hypotheses. An FDR procedure is statistically more powerful than the traditional procedures of Bonferroni and Familywise Error Rate when controlling for multiple comparisons. Furthermore, to account for the correlation in test statistics in MRI due to limited spatial resolution and data smoothing, we applied both a simple conservative modification of the FDR procedure and the effective number of resolution elements in the data.

\section{ACKNOWLEDGMENTS}

The authors thank the young women who participated in this study and our top-notch dedicated research assistants, Laura Kurzius, Willa Marquis, Colleen Doyle, Sophie Foss, Carlo Nati, Zachary Toth, and Kirwan Walsh, for dedicated help with participant engagement and all of the data collection.

\section{STATEMENT OF FINANCIAL SUPPORT}

This research was supported by the National Institute of Mental Health (National Institutes of Health, Bethesda, MD) MH093677 to C.M. and B.S.P.

Disclosure: The authors declare that there are no financial conflicts of interest.

\section{REFERENCES}

1. Stoltzfus RJ. Iron deficiency: global prevalence and consequences. Food Nutr Bull 2003;24:Suppl 4:S99-103.

2. Cao C, O’Brien KO. Pregnancy and iron homeostasis: an update. Nutr Rev 2013;71:35-51.

3. Derbyshire E, Davies GJ, Costarelli V, Dettmar PW. Habitual micronutrient intake during and after pregnancy in Caucasian Londoners. Matern Child Nutr 2009;5:1-9.

4. Siddappa AM, Rao R, Long JD, Widness JA, Georgieff MK. The assessment of newborn iron stores at birth: a review of the literature and standards for ferritin concentrations. Neonatology 2007;92:73-82.

5. Lozoff B, Georgieff MK. Iron deficiency and brain development. Semin Pediatr Neurol 2006;13:158-65.

6. Carlson ES, Tkac I, Magid R, et al. Iron is essential for neuron development and memory function in mouse hippocampus. J Nutr 2009;139:672-9.

7. Georgieff MK. Long-term brain and behavioral consequences of early iron deficiency. Nutr Rev 2011;69:Suppl 1:S43-8.

8. Fretham SJ, Carlson ES, Wobken J, Tran PV, Petryk A, Georgieff MK. Temporal manipulation of transferrin-receptor-1-dependent iron uptake identifies a sensitive period in mouse hippocampal neurodevelopment. Hippocampus 2012;22:1691-702.

9. Wu LL, Zhang L, Shao J, Qin YF, Yang RW, Zhao ZY. Effect of perinatal iron deficiency on myelination and associated behaviors in rat pups. Behav Brain Res 2008;188:263-70.

10. Yu GS, Steinkirchner TM, Rao GA, Larkin EC. Effect of prenatal iron deficiency on myelination in rat pups. Am J Pathol 1986;125:620-4.

11. Siddappa AM, Georgieff MK, Wewerka S, Worwa C, Nelson CA, Deregnier RA. Iron deficiency alters auditory recognition memory in newborn infants of diabetic mothers. Pediatr Res 2004;55:1034-41.

12. DeBoer T, Wewerka S, Bauer PJ, Georgieff MK, Nelson CA. Explicit memory performance in infants of diabetic mothers at 1 year of age. Dev Med Child Neurol 2005;47:525-31.

13. Riggins T, Miller NC, Bauer PJ, Georgieff MK, Nelson CA. Consequences of low neonatal iron status due to maternal diabetes mellitus on explicit memory performance in childhood. Dev Neuropsychol 2009;34:762-79.

14. Tamura T, Goldenberg RL, Hou J, et al. Cord serum ferritin concentrations and mental and psychomotor development of children at five years of age. J Pediatr 2002;140:165-70.

15. Amin SB, Orlando M, Wang $\mathrm{H}$. Latent iron deficiency in utero is associated with abnormal auditory neural myelination in $\geq 35$ weeks gestational age infants. J Pediatr 2013;163:1267-71. 


\section{Articles $\mid$ Monketal.}

16. Taylor PA, Jacobson SW, van der Kouwe A, et al. A DTI-based tractography study of effects on brain structure associated with prenatal alcohol exposure in newborns. Hum Brain Mapp 2015;36:170-86.

17. Broekman BF, Wang C, Li Y, et al.; GUSTO Study Group. Gestational age and neonatal brain microstructure in term born infants: a birth cohort study. PLoS One 2014;9:e115229.

18. Ou X, Andres A, Cleves MA, et al. Sex-specific association between infant diet and white matter integrity in 8-y-old children. Pediatr Res 2014;76:535-43.

19. Shao J, Lou J, Rao R, et al. Maternal serum ferritin concentration is positively associated with newborn iron stores in women with low ferritin status in late pregnancy. J Nutr 2012;142:2004-9.

20. Young MF, Pressman E, Foehr ML, et al. Impact of maternal and neonatal iron status on placental transferrin receptor expression in pregnant adolescents. Placenta 2010;31:1010-4.

21. The Centers for Disease Control and Prevention. Nutrition for Everyone: Basics: Iron and Iron Deficiency, 2011. (http://www.cdc.gov/nutrition/ everyone/basics/vitamins/iron.html.)

22. The Centers for Disease Control and Prevention. PNSS Health Indicators: Maternal Health Indicators, 2011. (http://www.cdc.gov/pednss/what_is/ pnss_health_indicators.htm\#Maternal\%20Health\%20Indicator.)

23. Swartz JR, Carrasco M, Wiggins JL, Thomason ME, Monk CS. Age-related changes in the structure and function of prefrontal cortex-amygdala circuitry in children and adolescents: a multi-modal imaging approach. Neuroimage 2014;86:212-20.

24. Mukherjee P, Miller JH, Shimony JS, et al. Diffusion-tensor MR imaging of gray and white matter development during normal human brain maturation. AJNR Am J Neuroradiol 2002;23:1445-56.

25. Ball G, Srinivasan L, Aljabar P, et al. Development of cortical microstructure in the preterm human brain. Proc Natl Acad Sci USA 2013;110: 9541-6.

26. Kroenke CD, Van Essen DC, Inder TE, Rees S, Bretthorst GL, Neil JJ. Microstructural changes of the baboon cerebral cortex during gestational development reflected in magnetic resonance imaging diffusion anisotropy. J Neurosci 2007;27:12506-15.

27. Hao X, Xu D, Bansal R, et al. Multimodal magnetic resonance imaging: the coordinated use of multiple, mutually informative probes to understand brain structure and function. Hum Brain Mapp 2013;34:253-71.

28. Tau GZ, Peterson BS. Normal development of brain circuits. Neuropsychopharmacology 2010;35:147-68.

29. Fretham SJ, Carlson ES, Georgieff MK. The role of iron in learning and memory. Adv Nutr 2011;2:112-21.

30. Kwik-Uribe CL, Gietzen D, German JB, Golub MS, Keen CL. Chronic marginal iron intakes during early development in mice result in persistent changes in dopamine metabolism and myelin composition. J Nutr 2000;130:2821-30.

31. Lee DL, Strathmann FG, Gelein R, Walton J, Mayer-Pröschel M. Iron deficiency disrupts axon maturation of the developing auditory nerve. J Neurosci 2012;32:5010-5.

32. Chockalingam UM, Murphy E, Ophoven JC, Weisdorf SA, Georgieff MK. Cord transferrin and ferritin values in newborn infants at risk for prenatal uteroplacental insufficiency and chronic hypoxia. J Pediatr 1987;111: 283-6.

33. Padilla N, Junqué C, Figueras F, et al. Differential vulnerability of gray matter and white matter to intrauterine growth restriction in preterm infants at 12 months corrected age. Brain Res 2014;1545:1-11.

34. Rose J, Butler EE, Lamont LE, Barnes PD, Atlas SW, Stevenson DK. Neonatal brain structure on MRI and diffusion tensor imaging, sex, and neurodevelopment in very-low-birthweight preterm children. Dev Med Child Neurol 2009;51:526-35.

35. Woolrich MW, Jbabdi S, Patenaude B, et al. Bayesian analysis of neuroimaging data in FSL. Neuroimage 2009;45:Suppl 1:S173-86.

36. Alexander DC, Barker GJ. Optimal imaging parameters for fiber-orientation estimation in diffusion MRI. Neuroimage 2005;27:357-67.

37. Christensen GE, Rabbitt RD, Miller MI. 3D brain mapping using a deformable neuroanatomy. Phys Med Biol 1994;39:609-18. 